\title{
Rosuvastatin attenuates the elevation in blood pressure induced by overexpression of human C-reactive protein
}

\author{
Xuguang $\mathrm{Li}^{1}$, Guangtian Yang${ }^{1}$, Gang Zhao ${ }^{1}$, Bin $\mathrm{Wu}^{1}$, Matthew L Edin ${ }^{2}$, Darryl C Zeldin ${ }^{2}$ and \\ Dao Wen Wang ${ }^{1}$
}

C-reactive protein (CRP) has been shown to function as an inflammatory factor to induce endothelial dysfunction and hypertension in rats. The anti-inflammatory effects of statins suggest that they may attenuate CRP-induced endothelial dysfunction and hypertension in Sprague-Dawley rats. Male Sprague-Dawley rats were injected with an adeno-associated virus (AAV) to induce overexpression of human CRP (AAV-hCRP) or green fluorescent protein (GFP) control (AAV-GFP). At 2 months after injection, rats were administered rosuvastatin by daily oral gavage $\left(10 \mathrm{mg} \mathrm{kg}^{-1}\right)$ for 2 additional months. Rosuvastatin administration attenuated the increased blood pressure and loss of vascular endothelial nitric oxide synthase expression in AAV-hCRP-treated rats, and $\mathbf{N}$-nitro-L-arginine methyl ester blocked its hypotensive effect. Rosuvastatin also activated phosphoinositide 3-kinases/Akt, and inhibited Rho kinase activity in aorta. Rosuvastatin reduced the production of reactive oxygen species through downregulation of nicotinamide adenine dinucleotide phosphate oxidase subunits, p22 phox and gp91 phox, and upregulation of superoxide dismutase 1 expression. Rosuvastatin attenuated the increase in blood pressure in AAV-hCRP-treated rats through endothelial protection and antioxidant effects. Our data reveals a novel mechanism through which statins may lower blood pressure.

Hypertension Research (2011) 34, 869-875; doi:10.1038/hr.2011.44; published online 12 May 2011

Keywords: C-reactive protein; endothelial nitric oxide synthase; reactive oxygen species; statin

\section{INTRODUCTION}

Inflammatory processes are important participants in the pathophysiology of hypertension. ${ }^{1,2} \mathrm{C}$-reactive protein (CRP) is an acute-phase reactant, which has traditionally been viewed and used as a systemic marker of inflammation. Numerous epidemiological studies have demonstrated that minor elevations in CRP levels are associated with increased risk of hypertension. ${ }^{3-5}$ More recently, Vongpatanasin et al. and our group ${ }^{6,7}$ demonstrated that overexpressed CRP could directly increase systolic blood pressure in rats and mice. Together, these results suggested that CRP is not only a marker of inflammation but also an important participant in the development of hypertension.

There is accumulating evidence that 3-hydroxy-3-methylglutaryl coenzyme A reductase inhibitors, or statins, exert numerous beneficial effects independent of their action on blood lipids. ${ }^{8}$ The beneficial cardiovascular mechanisms of statins include modulation of endothelial function, ${ }^{9,10}$ anti-inflammatory actions, ${ }^{11}$ and antioxidant properties. ${ }^{12}$ In the past few years, some studies have revealed that statins could decrease blood pressure by $>20 \mathrm{~mm} \mathrm{Hg}$ in spontaneously hypertensive rats. ${ }^{13,14}$

Patients receiving statin therapy in addition to antihypertensive drugs have a more pronounced reduction in blood pressure. ${ }^{15,16}$ Recently, the University of California, San Diego, Statin Study demon- strated that statins moderately but significantly reduced systolic blood pressure by $2.2 \mathrm{~mm} \mathrm{Hg}$ and diastolic blood pressure by $2.4 \mathrm{~mm} \mathrm{Hg}$ relative to placebo. ${ }^{17}$ These findings suggest that statin treatment could decrease blood pressure, though others have failed to reproduce this hypotensive effect. ${ }^{18}$ Several clinical trials, including the JUPITER study, ${ }^{19}$ have shown that statin therapy could significantly reduce CRP levels and improve clinical outcomes independent of effects on low-density lipoprotein cholesterol. ${ }^{11}$ Hence, the purpose of this was to investigate whether the rosuvastatin exerts antihypertensive effects and improves endothelial function and vascular oxidative stress in a rat model of CRP-overexpression-induced hypertension.

\section{METHODS}

Preparation of recombinant adeno-associated virus (AAV)

Type-8 double-stranded recombinant adeno-associated virus vectors (dsAAV8) containing either human CRP (AAV-hCRP) or green fluorescent protein (AAVGFP) cDNAs were prepared by triple plasmid co-transfection in HEK293 cells as described. ${ }^{6,20}$

\section{Animals}

Male Sprague-Dawley rats weighing 200-250g were obtained from the Institutional Animal Research Committee of Tongji Medical College and

\footnotetext{
${ }^{1}$ Departments of Internal Medicine, The Institute of Hypertension, Tongji Hospital, Tongji Medical College, Huazhong University of Science and Technology, Wuhan, PR China and ${ }^{2}$ Division of Intramural Research, National Institute of Environmental Health Sciences, NIH, Research Triangle Park, NC, USA

Correspondence: Dr DW Wang, Department of Internal Medicine, The Institute of Hypertension, Tongji Hospital, Tongji Medical College, Huazhong University of Science \& Technology, 1095\# Jiefang Avenue, Wuhan 430030, PR China.

E-mail: dwwang@tjh.tjmu.edu.cn

Received 13 November 2010; revised 10 February 2011; accepted 17 February 2011; published online 12 May 2011
} 
treated in compliance with the National Institutes of Health Guidelines for the Care and Use of Laboratory Animals. In all, 36 rats were randomized to three treatment groups (12 animals per group): saline control, AAV-GFP control and AAV-hCRP. Animals received a single intravenous injection of either saline or AAV $\left(1 \times 10^{12}\right.$ virions per rat), respectively. At 2 months after AAV injection, half of the rats of each group began rosuvastatin (Crestor, AstraZeneca, Macclesfield, Cheshire, England, UK) treatment at a dose of $10 \mathrm{mg} \mathrm{kg}^{-1}$ of body weight per day by oral gavage. Another half of the animals were given saline per day by oral gavage. Thus, there were six treatment groups, with six rats per group as follows: saline, saline+rosuvastatin, AAV-GFP, AAV-GFP+rosuvastatin, AAV-hCRP and AAV-hCRP+rosuvastatin. All animals were housed in a temperature and humidity controlled facility with 12-h light/dark cycles and allowed free access to normal rat chow and water ad libitum. Rats were killed at 4 months after injection after performing hemodynamic measurements. In addition, to investigate the mechanism of rosuvastatin against CRP-induced hypertension, 20 rats were divided to four groups: saline, AAVhCRP, AAV-CRP+rosuvastatin and AAV-CRP+rosuvastatin $+N$-nitro-L-arginine methyl ester (L-NAME). At 6 weeks after AAV injection, two groups began rosuvastatin treatment and L-NAME administration $\left(500 \mathrm{mgl}^{-1}\right.$ in drink water) for 6 weeks.

\section{Measurement of blood pressure and hemodynamics}

Systolic blood pressure was measured each month by the photoelectric tail-cuff method (Powerlab NIBP system, Bella Vista, NSW, Australia) in conscious animals. At the end of the experiment, heart rate, arterial elastance, maximum left ventricular pressure, cardiac output, rate of contraction $(\mathrm{dP} / \mathrm{dt} \max )$ and inter-arterial blood pressure were measured invasively using a Millar microtransducer catheter (SPR-838, Millar Instruments, Houston, TX, USA) as described previously. ${ }^{6}$

\section{Vasomotor function}

Thoracic aortas were rapidly isolated and cut into 4-mm segments. Aorta rings immersed in Krebs-Ringer $\mathrm{HCO}_{3}$ buffer, and responses to acetylcholine and sodium nitroprusside were assessed as previously described. ${ }^{21}$

\section{Measurement of superoxide release}

Aortas were cut into 5-mm segments and placed in Krebs-4-(2-hydroxyethyl)1-piperazineethanesulfonic acid buffer. Samples were transferred into scintillation vials that contained $2 \mathrm{ml}$ of Krebs-4-(2-hydroxyethyl)-1-piperazineethanesulfonic acid buffer with $5 \mathrm{mmoll}^{-1}$ lucigenin. Superoxide release was expressed as relative chemiluminescence per milligram of aortic tissue as previously described. ${ }^{22}$

\section{Immunoblotting}

Frozen tissues were sonicated in RIPA lysis buffer and protein concentrations were determined by the Bradford method. Extracts were resolved by SDSpolyacrylamide gel electrophoresis and transferred to polyvinylidene difluoride membranes. Proteins were visualized by immunoblotting with enhanced chemiluminescence (Pierce, Rockford, IL, USA) as previously described. ${ }^{23}$

\section{Statistical analysis}

Data are presented as mean \pm s.e.m. obtained in 3-12 independent analyses. Comparisons among groups were made by one-way analysis of variance, followed by a Scheffe F-test. $P<0.05$ was used to indicate statistical significance.

\section{RESULTS}

\section{Effect of AAV-hCRP treatment on CRP expression}

Serum hCRP levels were increased beginning at 1 month after AAVhCRP injection, and reached a peak at 4 months after injection. Rosuvastatin treatment did not significantly alter serum hCRP levels (Figure 1a). Immunoblots confirmed that rAAV-hCRP injection induced significant increases in hCRP expression in liver and aortic tissue after 4 months (Figure 1b). Thus, a single injection of AAVhCRP in rats induced significant and prolonged increases in circulating hCRP levels and hCRP tissue expression.

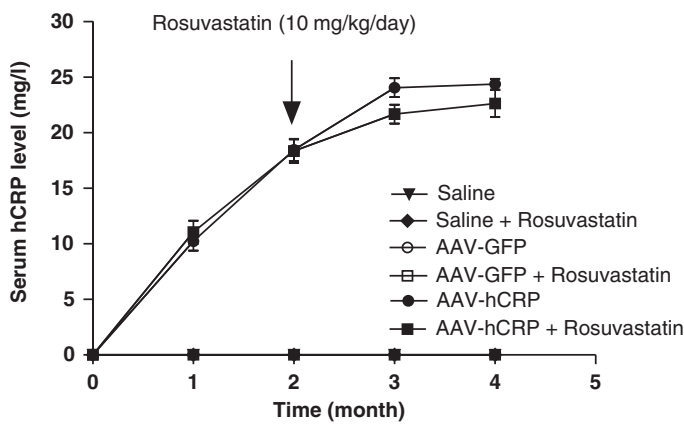

b

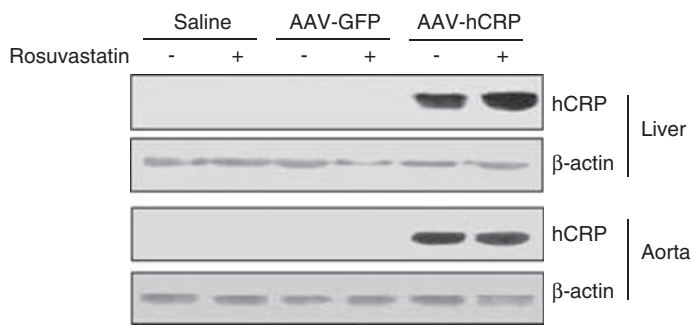

C

C Rosuvastatin (10 mg/kg/day)

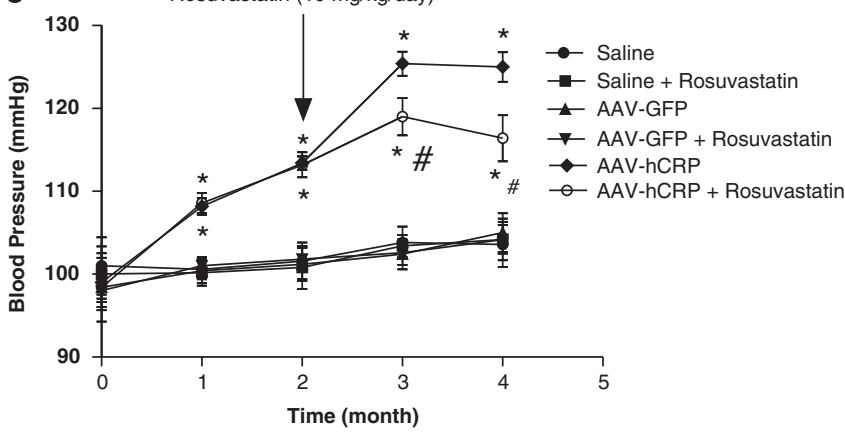

d

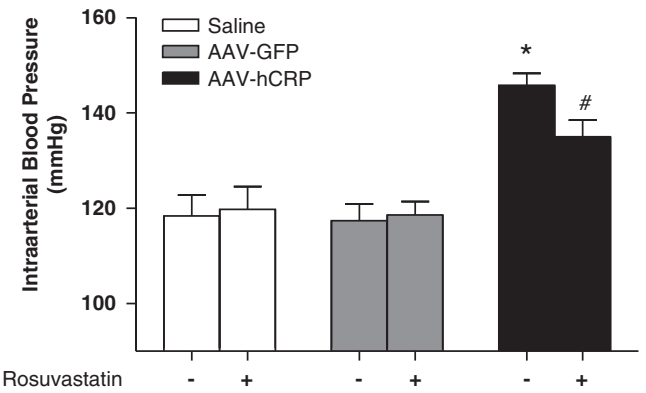

Figure 1 AAV-mediated expression of hCRP and effect of hCRP and rosuvastatin on systolic blood pressure. (a) Serum concentrations of hCRP at different times after injection of AAV vectors and rosuvastatin treatment. (b) Representative immunoblot of hCRP expression in liver and aorta at 4 months after gene delivery. (c) Systolic blood pressure was monitored from the beginning of the study using the tail-cuff method. Rats injected with AAV-hCRP had significantly increased blood pressure starting at 1 month after gene delivery $(P<0.05)$. Blood pressure was reduced by rosuvastatin treatment $(P<0.05)$. (d) Intra-arterial pressure was measured at the end of the experiment using the Millar catheter. Results were consistent with noninvasive blood pressure measurements $(P<0.05)$. Data represent mean \pm s.e.m. ${ }^{*} P<0.05$ vs. saline control group. ${ }^{\#} P<0.05$ vs. AAV-hCRP group, $(n=6)$.

\section{Effect of rosuvastatin on CRP-induced increases in systolic blood pressure}

Animals that received AAV-hCRP showed a significant increase in systolic blood pressure at 1 and 2 months after injection, an effect that 
became more pronounced ( $\sim 20 \mathrm{~mm} \mathrm{Hg}$ higher than control) at 3 and 4 months after injection (Figure 1c). The increased blood pressure in AAV-hCRP-treated rats was significantly attenuated by rosuvastatin treatment $(9 \mathrm{~mm} \mathrm{Hg}$ lower than AAV-hCRP group, $P<0.05$, Figure 1c). Intra-arterial blood pressure measurements were in agreement with non-invasive blood pressure measurements, and confirmed a significant increase following AAV-hCRP injection and an attenuation in animals treated with rosuvastatin $(P<0.05$, Figure 1d). Therefore, rosuvastatin modestly, but significantly, lowered systolic blood pressure induced by CRP overexpression in rats. Maximum left ventricular pressure, cardiac output and $\mathrm{dP} / \mathrm{dt} \max$ were not significantly altered after rosuvastatin treatment (Supplementary Table S1).

\section{Effect of rosuvastatin on arterial elastance and relaxation}

In agreement with our previous study, ${ }^{6} \mathrm{AAV}$-hCRP increased artery elastance and impaired vascular relaxation response to acetylcholine, which were significantly improved by rosuvastatin treatment $(P<0.05$, Figures $2 \mathrm{a}$ and $\mathrm{b}$ ), whereas vascular relaxation response to sodium
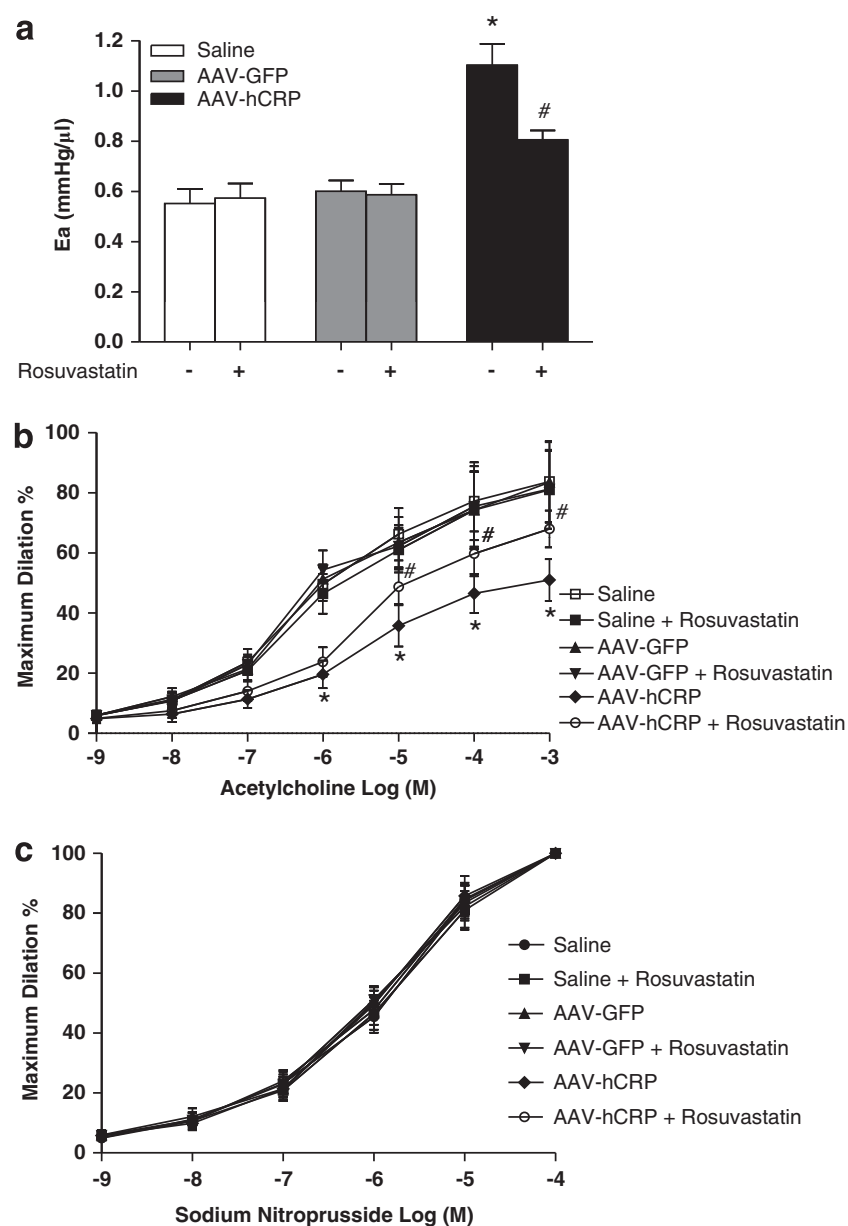

Figure 2 Effect of hCRP and rosuvastatin on vascular responsiness. (a) Arterial elastance was measured at the end of the experiment using the Millar catheter, AAV-hCRP increased arterial elastance, but rosuvastatin reversed it $(P<0.05)$. (b) Endothelial-dependent relaxation to acetylcholine in aortic segments. Rosuvastatin improved AAV-hCRP induced endothelia dysfunction $(P<0.05)$. (c) Endothelial-independent relaxation to sodium nitroprusside in aortic segments. There were no differences between groups. Data represent mean \pm s.e.m. ${ }^{*} P<0.05$ vs. saline control group. ${ }^{\#} P<0.05$ vs. AAV-hCRP group, $(n=4-6)$. nitroprusside was not altered by CRP and rosuvastatin therapy (Figure 2c). These results implicated rosuvastatin improved endothelial-dependent vascular relaxation response, which would contribute to lowering blood pressure.

\section{Effect of rosuvastatin on vascular endothelial signaling}

AAV-hCRP injection significantly reduced expression of endothelial nitric oxide synthase (eNOS) in aorta $(P<0.05$, Figures $3 \mathrm{a}$ and $\mathrm{b})$, consistent with our previous report. ${ }^{6}$ Phosphoinositide 3-kinases (PI3K) protein expression and Akt phosphorylation were also significantly reduced by AAV-hCRP injection. Interestingly, rosuvastatin treatment restored eNOS, PI3K and phosphorylated Akt to normal levels $(P<0.05$, Figures $3 \mathrm{a}$ and $\mathrm{b})$. Phosphorylation of myosinbinding subunit is a surrogate for Rho kinase (ROCK) activation, which contributes to arterial smooth muscle constriction. Interestingly, we found that myosin-binding subunit phosphorylation was increased by AAV-hCRP injection, an effect that was normalized by treatment with rosuvastatin $(P<0.05$, Figure $3 \mathrm{c})$. We also measured vascular nitrate/nitrite levels using a nitric oxide assay kit. Similar to effects on eNOS expression, rosuvastatin treatment attenuated the loss of vascular nitrate/nitrite observed with AAVhCRP injection $(P<0.05$, Figure $3 \mathrm{~d})$. Supplementary Figure $2 \mathrm{~S}$ shows that rosuvastatin treatment also attenuated the loss of serum nitrate/nitrite observed with AAV-hCRP injection. In additional animal experiment, L-NAME remarkably blocked hypotensive effect of rosuvastatin in AAV-hCRP-injected rats (118.3 $\pm 1.54 \mathrm{vs}$. $131.8 \pm 2.13 \mathrm{~mm} \mathrm{Hg}, P<0.05$, Figures $3 \mathrm{e}$ and $\mathrm{f}$ ). Together, these results suggest that rosuvastatin decrease blood pressure by improving endothelial function in AAV-hCRP-injected rats via restoration of normal eNOS.

Effect of rosuvastatin on vascular nicotinamide adenine dinucleotide phosphate (NADPH) oxidase expression and reactive oxygen species (ROS) production

CRP has been shown to increase NADPH oxidase subunit expression and ROS production in vascular cells and vasculature. ${ }^{24-26}$ Therefore, we investigated whether CRP or rosuvastatin influenced vascular NADPH oxidase subunit expression and/or vascular ROS production. AAV-hCRP injection markedly induced expression of NADPH oxidase p22 phox and gp91 phox subunits, an effect that was reversed by rosuvastatin treatment $(P<0.05$, Figures $4 \mathrm{a}$ and $\mathrm{b})$. Expression of p40 phox, p47 phox and p67 phox were not significantly altered by AAV-hCRP injection or rosuvastatin treatment (Figure 4a). Levels of superoxide dismutase 1 (SOD-1), an important antioxidant enzyme, were diminished by AAV-hCRP injection, an effect that was normalized by rosuvastatin treatment $(P<0.05$, Figures $4 \mathrm{a}$ and $\mathrm{b})$. Vascular production of ROS was assessed by a lucigenin chemiluminescence assay in isolated aortic segments. AAV-hCRP injection increased vascular production of superoxide by two- to threefold, an effect that was significantly attenuated by rosuvastatin treatment $(P<0.05$, Figure 4c). Together, these results suggest that CRP overexpression upregulated p22 phox and gp91 phox expression, which led to increased vascular ROS production. CRP overexpression also may have led to increased ROS through downregulation of the antioxidant enzyme SOD-1. Rosuvastatin attenuated the reduction in SOD-1 and restored ROS levels to normal. Similar to our previous studies, vascular angiotensin $1\left(\mathrm{AT}_{1}\right)$ receptor expression was upregulated and $\mathrm{AT}_{2}$ receptor expression was downregulated in AAV-hCRPinjected rats. Rosuvastatin treatment attenuated the rise in vascular $\mathrm{AT}_{1}$ receptor expression but did not alter $\mathrm{AT}_{2}$ receptor expression (Supplementary Figure S1). 
a

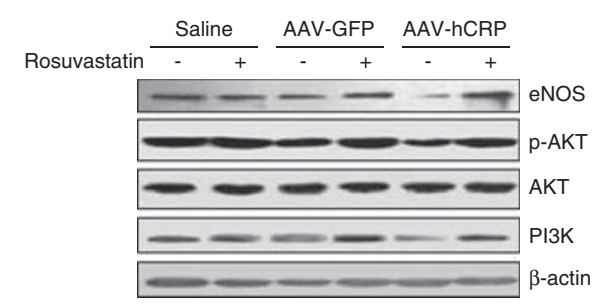

C
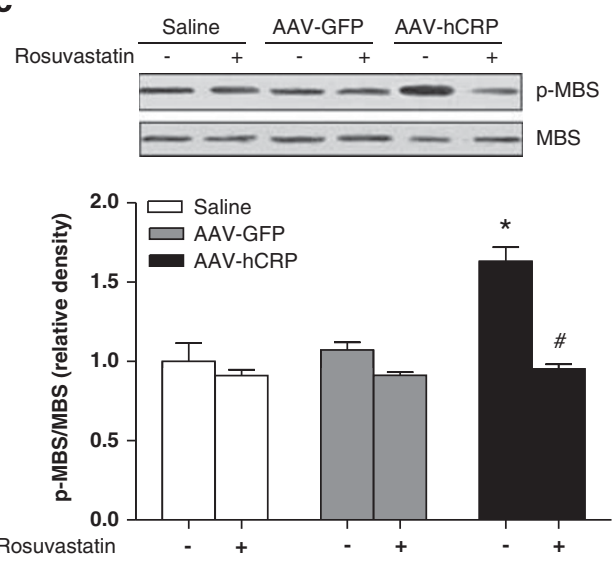

e

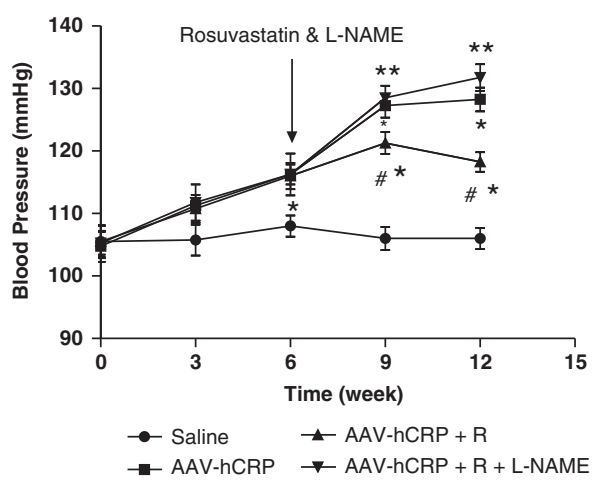

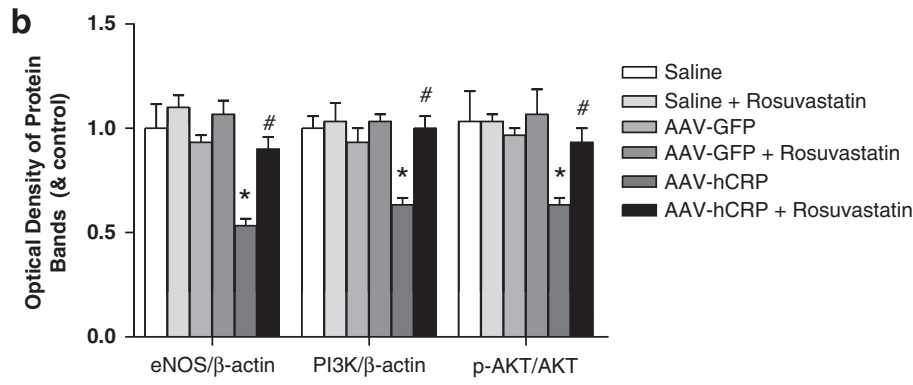

d

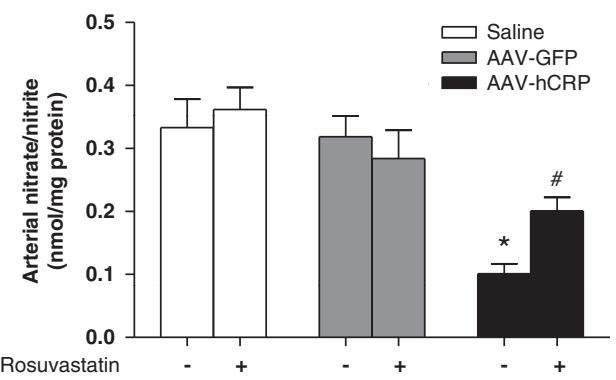

$f$

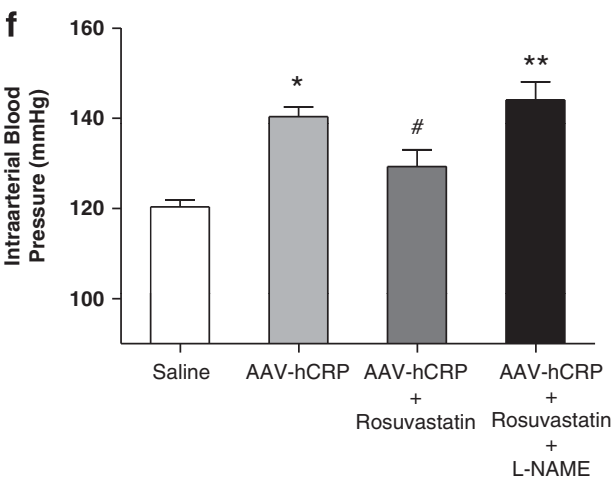

Figure 3 Effect of hCRP and rosuvastatin on endothelial function. (a) Representative immunoblot analysis for eNOS, PI3K and Akt. (b) Densitometric quantification shows eNOS expression, PI3K expression and Akt phosphorylation were suppressed by AAV-hCRP but normalized by rosuvastatin treatment $(P<0.05)$; (c) Immunoblot and relative densitometric analysis show that myosin-binding substrate (MBS) phosphorylation was increased by AAV-hCRP injection, but normalized by rosuvastatin treatment $(P<0.05)$. (d) AAV-hCRP injection induced a decrease in vascular nitrate/nitrite concentration, an effect that was attenuated by rosuvastatin treatment $(P<0.05)$. (e, f) L-NAME blocked hypotensive effect of rosuvastatin $(P<0.05)$. Data represent mean \pm s.e.m. ${ }^{*} P<0.05$ vs. saline control group. ${ }^{\#} P<0.05$ vs. AAV-hCRP group. ${ }^{*} P<0.05$ vs. AAV-hCRP+rosuvastatin, $(n=3-4)$.

Effect of rosuvastatin on CRP-induced c-Jun N-terminal kinase (JNK) and mitogen-activated protein kinase (MAPK) activation CRP has been shown to activate JNK and MAPK in vascular smooth muscle cells and endothelial cells. ${ }^{27-29}$ p38 MAPK have been shown to be activated by CRP and mediate vascular ROS production induced by CRP. ${ }^{25}$ To investigate whether rosuvastatin modifies these signaling pathways in the vasculature, we evaluated extracellular signal-regulated kinase (ERK) 1/2, p38 MAPK and JNK activation. AAV-hCRP injection significantly induced ERK1/2-and p38 MAPK phosphorylation in aorta $(P<0.05$, Figures $5 \mathrm{a}$ and $\mathrm{b})$. The effects of CRP overexpression on ERK1/2 and p38 MAPK phosphorylation were attenuated by rosuvastatin treatment (Figures $5 \mathrm{a}$ and $\mathrm{b}$ ).

\section{Physiological parameters of animals}

Plasma concentrations of total, high-density lipoprotein and lowdensity lipoprotein cholesterol, and triglycerides at 4 months after
AAV-hCRP injection and at 2 months after rosuvastatin treatment are shown in Table 1. All measured parameters were within the normal range in the control group. Neither rosuvastatin treatment nor AAV-hCRP injection significantly altered plasma concentrations of cholesterol or triglycerides.

\section{DISCUSSION}

AAV delivery of hCRP resulted in long term, stable hCRP expression, induced markers of vascular dysfunction, and increased systolic blood pressure consistent with previous studies. ${ }^{6,7}$ This study demonstrates that rosuvastatin treatment significantly attenuates the hypertension and dysregulation of vascular signaling induced by CRP.

Our initial hypothesis was that rosuvastatin treatment might lower blood pressure by reducing high serum CRP level. Previous studies show that CRP is mainly synthesized in the liver and secreted into the systemic circulation, and suggest that statins may reduce CRP levels by 


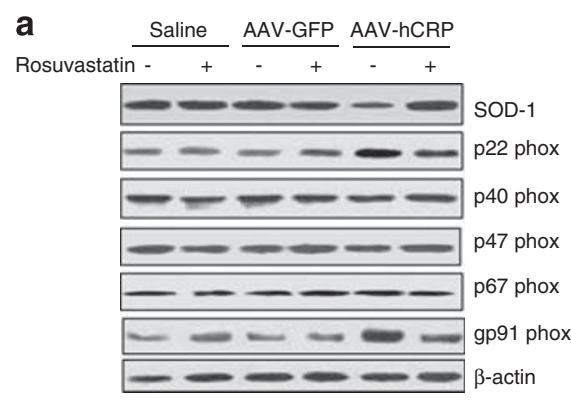

C
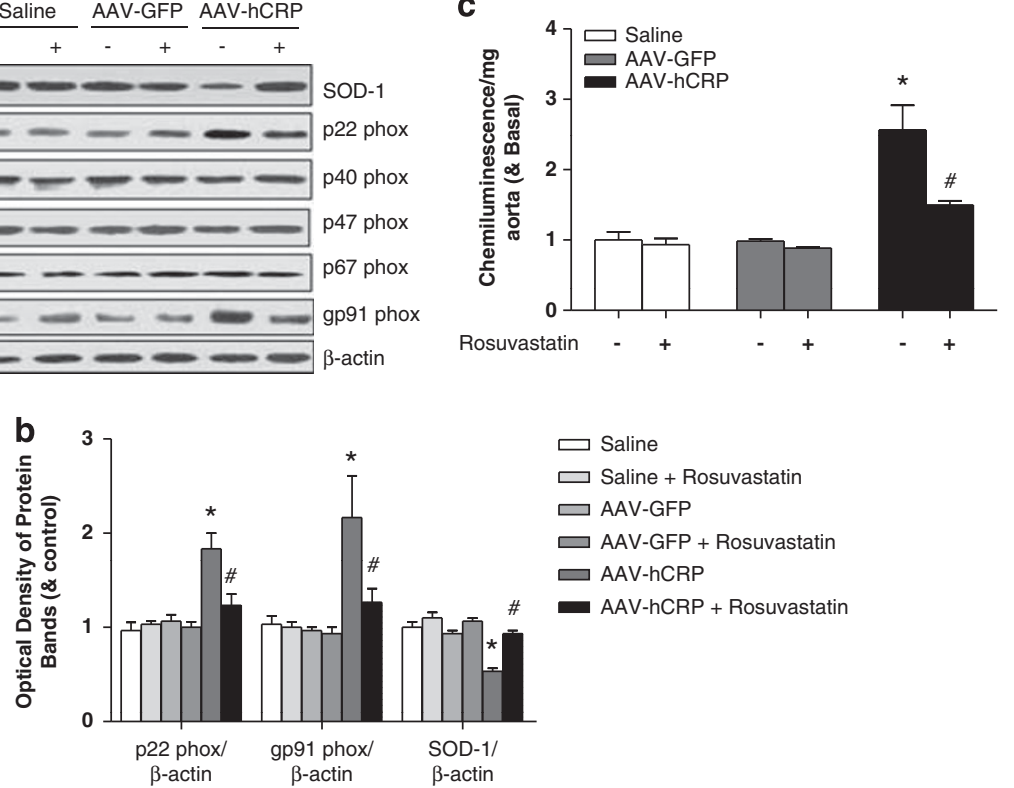

Figure 4 Effect of hCRP and rosuvastatin on vascular expression NAD(P)H oxidase subunits and SOD-1. (a) Representative immunoblot analysis for p22 phox, p40 phox, p47 phox, p67 phox, gp91 phox and SOD-1 expression in vasculature. (b) Densitometric analysis of immunoblots shows that CRP-induced increases in $\mathrm{p} 22$ phox and gp91phox expression were abolished by rosuvastatin treatment $(P<0.05)$. SOD-1 was downregulated by AAV-hCRP injection, but normalized by rosuvastatin treatment $(P<0.05)$. (c) Superoxide release is expressed as relative chemiluminescence per milligram of aortic tissue (control=100; $P<0.05$ ). Data represent mean \pm s.e.m. ${ }^{*} P<0.05$ vs. saline control group. ${ }^{\#} P<0.05$ vs. AAV-hCRP group, $(n=3-4)$.

a
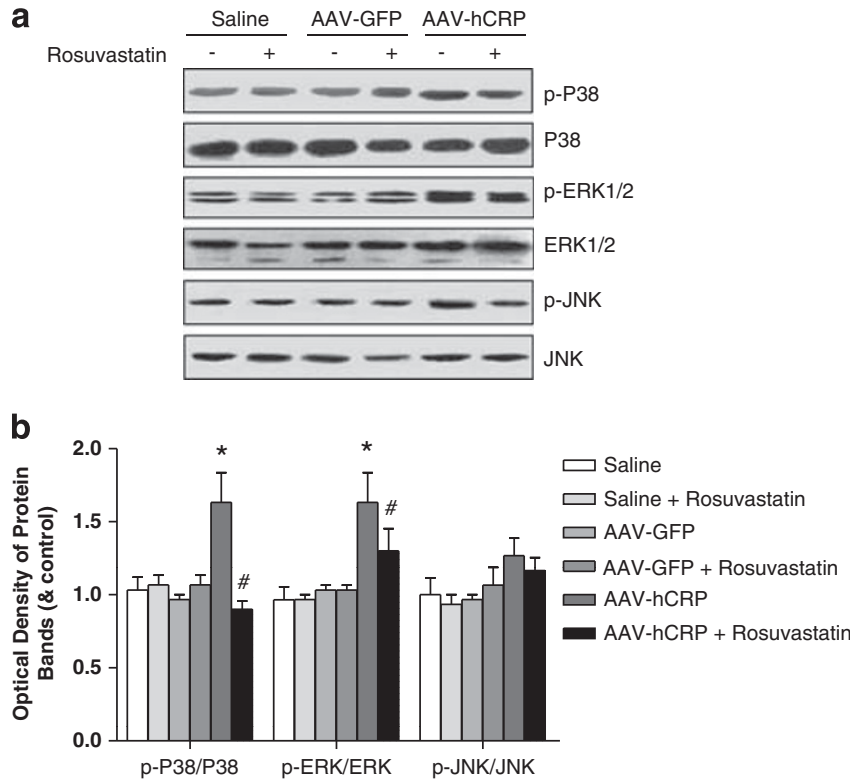

Figure 5 Effect of hCRP and rosuvastatin on vascular MAPK activation. (a) Representative immunoblot of ERK1/2, p38 MAPK and JNK. (b) Vascular p38 MAPK and ERK1/2 phosphorylation were increased in AAVhCRP-injected rats, an effect that was attenuated by rosuvastatin $(P<0.05)$. Results are expressed as the ratio of phospho-ERK1/2, p38 and JNK to total ERK1/2, p38, JNK, respectively. Data represent mean \pm s.e.m. ${ }^{*} P<0.05$ vs. saline control group. ${ }^{\#} P<0.05$ vs. AAV-hCRP group, $(n=3-4)$.

decreasing CRP synthesis in hepatocytes. ${ }^{30}$ However, AAV-hCRP induced prolonged and sustained gene expression, which was independent on excretion from hepatocytes, so that circulating CRP levels was not reduced by rosuvastatin. Systolic blood pressure was increased after AAV-CRP administration. While not lowering CRP levels significantly, rosuvastatin decreased blood pressure significantly. In contrast, blood pressure in control and AAV-GFP groups were not elevated after AAV-hCRP injection or altered by rosuvastatin treatment. Our results are not consistent with some previous reports, ${ }^{13,14}$ which may be because of the differences in statistical power, experimental conditions or animal models used in these studies.

Arterial elastance is a marker of aortic stiffness, and aortic stiffness was found to be an important determinant of future increases in blood pressure. ${ }^{31}$ CRP induced vasodilatory dysfunction and increased artery elastance, which were consistent with previous study. ${ }^{6,25}$ The partial reverse of CRP-induced hypertension by rosuvastatin was accompanied by a restoration of normal vascular relaxation and arterial elastance. CRP induced several changes indicative of endothelial dysfunction. AAV-hCRP induced a loss of eNOS expression and activity, likely through reduced PI3K and Akt activation, and increased Rho kinase activation. CRP also upregulated $\mathrm{AT}_{1}$ receptor expression and NADPH oxidase subunit expression, while downregulating SOD-1, and led to an increase in vascular ROS production. Rosuvastatin treatment produced a nearly complete reversal of these CRPinduced signaling changes. Thus, statin therapy may reduce hypertension through a variety of signaling mechanisms, which reverse vascular dysfunction.

Endothelial-derived NO is an important regulator of blood pressure. ${ }^{32}$ Our previous studies suggested that CRP-induced hypertension may be due to impairment of eNOS expression and activity. ${ }^{6}$ In this study, statins can affect the expression and activity of eNOS downstream of PI3K/Akt and ROCK activation, and improved eNOS activity and nitric oxide production, which is likely a primary cause of the blood pressure reduction. Furthermore, rosuvastatin failed to decrease blood pressure in L-NAME-treated rats, which was consistent with previous study. ${ }^{13}$ Therefore, rosuvastatin therapy reverses the loss of PI3K expression and Akt activity, and the increase in ROCK activation seen in AAV-hCRP-treated rats. By preventing the 
Table 1 Lipid profile in control and rosuvastatin-treated rats at 4 months after AAV treatment

\begin{tabular}{|c|c|c|c|c|}
\hline & Cholesterol (mg dl-1) & $H D L\left(m g d l^{-1}\right)$ & $L D L\left(m g d l^{-1}\right)$ & Triglyceride (mg dl-1) \\
\hline Saline+rosuvastatin & $41.8 \pm 1.4$ & $18.4 \pm 1.7$ & $18.6 \pm 0.9$ & $27.2 \pm 2.3$ \\
\hline AAV-GFP+rosuvastatin & $42.2 \pm 2.7$ & $19.2 \pm 2.1$ & $19.6 \pm 1.4$ & $27.6 \pm 2.4$ \\
\hline AAV-hCRP & $42.4 \pm 3.2$ & $17.0 \pm 1.5$ & $18.2 \pm 1.0$ & $29.0 \pm 1.9$ \\
\hline AAV-hCRP+rosuvastatin & $41.6 \pm 2.1$ & $16.6 \pm 2.1$ & $17.6 \pm 1.5$ & $28.4 \pm 1.5$ \\
\hline
\end{tabular}

Abbreviations: AAV, adeno-associated virus; GFP, green fluorescent protein; hCRP, human C-reactive protein; LDL, low-density protein; HDL, high-density protein.

Plasma concentrations of total, LDL and HDL cholesterol, and triglycerides were determined at 4 months after AAV-hCRP injection and at 2 months after rosuvastatin treatment. Data are expressed as mean \pm s.e.m. ( $n=6$ per group). There were no significant differences between the treatment groups.

dysregulation of these pathways, rosuvastatin may maintain normal eNOS signaling in the vasculature. Rho kinases are also recognized as major regulators of cell contraction, via myosin-binding subunit phosphorylation and inhibition of myosin light chain phosphatase activity. ${ }^{33}$ Statins have been shown to be efficient inhibitors of ROCKs ${ }^{10}$ and thus may present a novel therapeutic target in hypertension. ${ }^{34}$ Rho/Rho-kinase signaling has been shown to be activated in endothelial cells by CRP and mediate CRP-induced vascular ROS, ${ }^{35-37}$ as it did in aortas from our AAV-hCRP-treated rats. However, after 2 months of rosuvastatin therapy, ROCK activation was restored to normal levels in CRP-treated rat aorta, which would decrease vascular constriction and help reduce hypertension.

Tetrahydrobiopterin, a critical cofactor for eNOS, is synthesized from guanosine-triphosphate via de novo pathway by guanosinetriphosphate cyclohydrolase I enzyme. CRP has been shown to impair eNOS activity due to either increased reactive oxygen species or decreased tetrahydrobiopterin availability resulting in eNOS uncoupling. ${ }^{26}$ Besides that, a new study reported that CRP significantly increased eNOS binding to caveolin-1 and decreased binding to Hsp90 and porin, ${ }^{38}$ leading to endothelial dysfunction. Therefore, in this study, we cannot exclude the possibility that the effects of rosuvastatin were mediated via these potential mechanisms.

The relationship between oxidative stress and hypertension has been established in prior studies. ${ }^{39} \mathrm{NADPH}$ oxidases were a major source of ROS in the vascular system. ${ }^{40}$ The activation of vascular NADPH oxidase is dependent on the assembly of its membrane bound (gp91 phox and p22 phox) and cytoplasmic (p40 phox, p47 phox and p67 phox) subunits. ${ }^{41}$ Similar to previous studies, ${ }^{25,26,37}$ CRP elevated ROS production correlated with increased $\mathrm{p} 22$ phox and gp 91 phox protein expression. Induction of $\mathrm{p} 22$ phox, gp 91 phox and ROS was attenuated by rosuvastatin treatment. These results are similar to those observed using rosuvastatin therapy in Ren2 transgenic rats. ${ }^{42}$ Rosuvastatin did not significantly affect $\mathrm{AT}_{2}$ receptor expression, whereas atorvastatin blocked p22 phox, but not gp91 phox upregulation. ${ }^{43}$ In addition to excess ROS generation, dysfunctional antioxidant defense mechanisms contribute to oxidative stress in hypertension. Superoxide dismutases represent a major antioxidant defense system in the vasculature. The $\mathrm{Cu} / \mathrm{Zn}$ cytosolic SOD (SOD-1) is highly expressed in the endothelium. ${ }^{44}$ SOD-1 was diminished by CRP but restored by rosuvastatin, in agreement with previous observations in bovine aortic endothelial cells. ${ }^{45}$ Moreover, CRP was demonstrated to inhibited eNOS activity through increasing ROS production in endothelial cells. ${ }^{26}$ Thus, increased antioxidant defenses and diminished NADPH oxidase expression on rosuvastatin therapy might compensate deterioration of eNOS expression and endothelial function, which may contribute to lower blood pressure. In vascular smooth muscle cells, $\mathrm{AT}_{1}$ receptor expression and $\mathrm{AT}_{1}$-mediated production of ROS were increased by CRP, ${ }^{24}$ thus, decreased vascular
$\mathrm{AT}_{1}$ receptor expression may also result in reduced ROS production and hypertension. ERK1/2, p38 MAPK and JNK are activated by CRP in vascular endothelial cells and vascular smooth muscle cells. ${ }^{27-29}$ In addition, p38 MAPK have been shown to be mediated vascular ROS production induced by CRP, ${ }^{25}$ p 38 MAPK, ERK $1 / 2$ and JNK have been shown to promote vascular smooth muscle contraction and vascular fibrosis, ${ }^{46-49}$ which may promote development of hypertension. Rosuvastatin therapy reversed p38 MAPK activation in vasculature, which may contribute to reduced ROS production. ${ }^{25}$ Decreased ERK activation on rosuvastatin treatment might improve vascular remodeling. ${ }^{43}$ In summary, rosuvastatin therapy inhibited p38 MAPK and ERK activation may also have contributed to reduced hypertension. In conclusion, these results demonstrate that rosuvastatin attenuates CRP-induced hypertension through improvement of vascular endothelial function and reduction of oxidative stress. These results also support the potential for the use of statins as an additive therapy for the treatment of resistant hypertension associated with increased CRP.

\section{CONFLICT OF INTEREST}

The authors declare no conflict of interest.

\section{ACKNOWLEDGEMENTS}

This work was supported by '973' project (no. 2007CB512004), Wuhan City grants and Hubei Province project on risk prediction of Cardiovascular Diseases and the Intramural Research Program of the NIH, National Institute of Environmental Health Sciences (Z01 ES025043).

1 Grundy SM. Inflammation, hypertension, and the metabolic syndrome. JAMA 2003; 290: 3000-3002.

2 Savoia C, Schiffrin EL. Inflammation in hypertension. Curr Opin Nephrol Hypertens 2006; 15: 152-158.

3 Lakoski SG, Cushman M, Palmas W, Blumenthal R, D’Agostino Jr RB, Herrington DM. The relationship between blood pressure and C-reactive protein in the Multi-Ethnic Study of Atherosclerosis (MESA). J Am Coll Cardiol 2005; 46: 1869-1874.

4 Sesso HD, Buring JE, Rifai N, Blake GJ, Gaziano JM, Ridker PM. C-reactive protein and the risk of developing hypertension. JAMA 2003; 290: 2945-2951.

5 Sung KC, Suh JY, Kim BS, Kang JH, Kim H, Lee MH, Park JR, Kim SW. High sensitivity $\mathrm{C}$-reactive protein as an independent risk factor for essential hypertension. Am J Hypertens 2003; 16: 429-433.

6 Guan H, Wang P, Hui R, Edin ML, Zeldin DC, Wang DW. Adeno-associated virusmediated human $\mathrm{C}$-reactive protein gene delivery causes endothelial dysfunction and hypertension in rats. Clin Chem 2009; 55: 274-284.

7 Vongpatanasin W, Thomas GD, Schwartz R, Cassis LA, Osborne-Lawrence S, Hahner L, Gibson LL, Black S, Samols D, Shaul PW. C-reactive protein causes downregulation of vascular angiotensin subtype 2 receptors and systolic hypertension in mice. Circulation 2007; 115: 1020-1028.

8 Auer J, Berent R, Weber T, Eber B. Clinical significance of pleiotropic effects of statins: lipid reduction and beyond. Curr Med Chem 2002; 9: 1831-1850.

9 Laufs U, La Fata V, Plutzky J, Liao JK. Upregulation of endothelial nitric oxide synthase by HMG CoA reductase inhibitors. Circulation 1998; 97: 1129-1135.

10 Wolfrum S, Jensen KS, Liao JK. Endothelium-dependent effects of statins. Arterioscler Thromb Vasc Biol 2003; 23: 729-736. 
11 Ridker PM, Cannon CP, Morrow D, Rifai N, Rose LM, McCabe CH, Pfeffer MA, Braunwald E. C-reactive protein levels and outcomes after statin therapy. $N$ Engl J Med 2005; 352: 20-28.

12 Wassmann S, Laufs U, Muller K, Konkol C, Ahlbory K, Baumer AT, Linz W, Bohm M, Nickenig G. Cellular antioxidant effects of atorvastatin in vitro and in vivo. Arterioscler Thromb Vasc Biol 2002; 22: 300-305.

13 Susic D, Varagic J, Ahn J, Slama M, Frohlich ED. Beneficial pleiotropic vascular effects of rosuvastatin in two hypertensive models. J Am Coll Cardiol 2003; 42: 1091-1097.

14 Wassmann S, Laufs U, Baumer AT, Muller K, Ahlbory K, Linz W, Itter G, Rosen R, Bohm $\mathrm{M}$, Nickenig G. HMG-CoA reductase inhibitors improve endothelial dysfunction in normocholesterolemic hypertension via reduced production of reactive oxygen species. Hypertension 2001; 37: 1450-1457.

15 Borghi C, Prandin MG, Costa FV, Bacchelli S, Degli Esposti D, Ambrosioni E. Use of statins and blood pressure control in treated hypertensive patients with hypercholesterolemia. J Cardiovasc Pharmacol 2000; 35: 549-555.

16 Glorioso N, Troffa C, Filigheddu F, Dettori F, Soro A, Parpaglia PP, Collatina S, Pahor M. Effect of the HMG-CoA reductase inhibitors on blood pressure in patients with essential hypertension and primary hypercholesterolemia. Hypertension 1999; 34: 1281-1286.

17 Golomb BA, Dimsdale JE, White HL, Ritchie JB, Criqui MH. Reduction in blood pressure with statins: results from the UCSD Statin Study, a randomized trial. Arch Intern Med 2008; 168: 721-727.

18 Williams B, Lacy PS, Cruickshank JK, Collier D, Hughes AD, Stanton A, Thom S, Thurston $\mathrm{H}$. Impact of statin therapy on central aortic pressures and hemodynamics: principal results of the Conduit Artery Function Evaluation-Lipid-Lowering Arm (CAFELLA) Study. Circulation 2009; 119: 53-61.

19 Ridker PM, Danielson E, Fonseca FA, Genest J, Gotto Jr AM, Kastelein JJ, Koenig W, Libby P, Lorenzatti AJ, MacFadyen JG, Nordestgaard BG, Shepherd J, Willerson JT, Glynn RJ. Rosuvastatin to prevent vascular events in men and women with elevated Creactive protein. N Engl J Med 2008; 359: 2195-2207.

20 Xiao X, Li J, Samulski RJ. Production of high-titer recombinant adeno-associated virus vectors in the absence of helper adenovirus. J Virol 1998; 72: 2224-2232.

21 Xiao B, Li X, Yan J, Yu X, Yang G, Xiao X, Voltz JW, Zeldin DC, Wang DW. Overexpression of cytochrome P450 epoxygenases prevents development of hypertension in spontaneously hypertensive rats by enhancing atrial natriuretic peptide. J Pharmacol Exp Ther 2010; 334: 784-794.

22 Somers MJ, Mavromatis K, Galis ZS, Harrison DG. Vascular superoxide production and vasomotor function in hypertension induced by deoxycorticosterone acetate-salt. Circulation 2000; 101: 1722-1728.

23 Jiang JG, Chen CL, Card JW, Yang S, Chen JX, Fu XN, Ning YG, Xiao X, Zeldin DC, Wang DW. Cytochrome P450 2J2 promotes the neoplastic phenotype of carcinoma cells and is up-regulated in human tumors. Cancer Res 2005; 65: 4707-4715.

24 Wang CH, Li SH, Weisel RD, Fedak PW, Dumont AS, Szmitko P, Li RK, Mickle DA, Verma S. C-reactive protein upregulates angiotensin type 1 receptors in vascular smooth muscle. Circulation 2003; 107: 1783-1790.

25 Nagaoka T, Kuo L, Ren Y, Yoshida A, Hein TW. C-reactive protein inhibits endotheliumdependent nitric oxide-mediated dilation of retinal arterioles via enhanced superoxide production. Invest Ophthalmol Vis Sci 2008; 49: 2053-2060.

26 Singh U, Devaraj S, Vasquez-Vivar J, Jialal I. C-reactive protein decreases endothelial nitric oxide synthase activity via uncoupling. J Mol Cell Cardiol 2007; 43: 780-791.

27 Hattori Y, Matsumura M, Kasai K. Vascular smooth muscle cell activation by C-reactive protein. Cardiovasc Res 2003; 58: 186-195.

28 Qamirani E, Ren Y, Kuo L, Hein TW. C-reactive protein inhibits endothelium-dependent NO-mediated dilation in coronary arterioles by activating p38 kinase and $\mathrm{NAD}(\mathrm{P}) \mathrm{H}$ oxidase. Arterioscler Thromb Vasc Biol 2005; 25: 995-1001.

29 Wu J, Stevenson MJ, Brown JM, Grunz EA, Strawn TL, Fay WP. C-reactive protein enhances tissue factor expression by vascular smooth muscle cells: mechanisms and in vivo significance. Arterioscler Thromb Vasc Biol 2008; 28: 698-704.

30 Arnaud C, Burger F, Steffens S, Veillard NR, Nguyen TH, Trono D, Mach F. Statins reduce interleukin-6-induced $C$-reactive protein in human hepatocytes: new evidence for direct antiinflammatory effects of statins. Arterioscler Thromb Vasc Biol 2005; 25: 1231-1236.
31 Dernellis J, Panaretou M. Aortic stiffness is an independent predictor of progression to hypertension in nonhypertensive subjects. Hypertension 2005; 45: 426-431.

32 Huang PL, Huang Z, Mashimo H, Bloch KD, Moskowitz MA, Bevan JA, Fishman MC. Hypertension in mice lacking the gene for endothelial nitric oxide synthase. Nature 1995; 377: 239-242.

33 Uehata M, Ishizaki T, Satoh H, Ono T, Kawahara T, Morishita T, Tamakawa H, Yamagami K, Inui J, Maekawa M, Narumiya S. Calcium sensitization of smooth muscle mediated by a Rho-associated protein kinase in hypertension. Nature 1997; 389: 990-994.

34 Mukai Y, Shimokawa H, Matoba T, Kandabashi T, Satoh S, Hiroki J, Kaibuchi K, Takeshita A. Involvement of Rho-kinase in hypertensive vascular disease: a novel therapeutic target in hypertension. FASEB J 2001; 15: 1062-1064.

35 Nakakuki T, Ito M, Iwasaki H, Kureishi Y, Okamoto R, Moriki N, Kongo M, Kato S, Yamada N, Isaka N, Nakano T. Rho/Rho-kinase pathway contributes to C-reactive protein-induced plasminogen activator inhibitor-1 expression in endothelial cells. Arterioscler Thromb Vasc Biol 2005; 25: 2088-2093.

36 Xu JW, Morita I, Ikeda K, Miki T, Yamori Y. C-reactive protein suppresses insulin signaling in endothelial cells: role of spleen tyrosine kinase. Mol Endocrinol 2007; 21: 564-573.

37 Kobayashi S, Inoue N, Ohashi Y, Terashima M, Matsui K, Mori T, Fujita H, Awano K, Kobayashi K, Azumi H, Ejiri J, Hirata K, Kawashima S, Hayashi Y, Yokozaki H, Itoh H, Yokoyama M. Interaction of oxidative stress and inflammatory response in coronary plaque instability: important role of C-reactive protein. Arterioscler Thromb Vasc Biol 2003; 23: 1398-1404

38 Valleggi S, Devaraj S, Dasu MR, Jialal I. C-reactive protein adversely alters the proteinprotein interaction of the endothelial isoform of nitric oxide synthase. Clin Chem 2010; 56: $1345-1348$

39 Paravicini TM, Touyz RM. NADPH oxidases, reactive oxygen species, and hypertension: clinical implications and therapeutic possibilities. Diabetes Care 2008; 31(Suppl 2): S170-S180

40 Bedard K, Krause KH. The NOX family of ROS-generating NADPH oxidases: physiology and pathophysiology. Physiol Rev 2007; 87: 245-313.

41 Lassegue B, Clempus RE. Vascular NAD(P)H oxidases: specific features, expression, and regulation. Am J Physiol Regul Integr Comp Physiol 2003; 285: R277-R297.

42 Habibi J, Whaley-Connell A, Qazi MA, Hayden MR, Cooper SA, Tramontano A, Thyfault J, Stump C, Ferrario C, Muniyappa R, Sowers JR. Rosuvastatin, a 3-hydroxy-3methylglutaryl coenzyme a reductase inhibitor, decreases cardiac oxidative stress and remodeling in Ren2 transgenic rats. Endocrinology 2007; 148: 2181-2188.

43 Briones AM, Rodriguez-Criado N, Hernanz R, Garcia-Redondo AB, Rodrigues-Diez RR, Alonso MJ, Egido J, Ruiz-Ortega M, Salaices M. Atorvastatin prevents angiotensin IIinduced vascular remodeling and oxidative stress. Hypertension 2009; 54: 142-149.

44 Faraci FM, Didion SP. Vascular protection: superoxide dismutase isoforms in the vessel wall. Arterioscler Thromb Vasc Biol 2004; 24: 1367-1373.

45 Desjardins F, Sekkali B, Verreth W, Pelat M, De Keyzer D, Mertens A, Smith G, Herregods MC, Holvoet P, Balligand JL. Rosuvastatin increases vascular endothelial PPARgamma expression and corrects blood pressure variability in obese dyslipidaemic mice. Eur Heart J 2008; 29: 128-137.

46 Kim B, Kim J, Bae YM, Cho SI, Kwon SC, Jung JY, Park JC, Ahn HY. p38 mitogenactivated protein kinase contributes to the diminished aortic contraction by endothelin1 in DOCA-salt hypertensive rats. Hypertension 2004; 43: 1086-1091.

47 Lee YR, Lee CK, Park HJ, Kim H, Kim J, Lee KS, Lee YL, Min KO, Kim B. c-Jun N-terminal kinase contributes to norepinephrine-induced contraction through phosphorylation of caldesmon in rat aortic smooth muscle. J Pharmacol Sci 2006; 100: 119-125.

48 Touyz RM, El Mabrouk M, He G, Wu XH, Schiffrin EL. Mitogen-activated protein/ extracellular signal-regulated kinase inhibition attenuates angiotensin II-mediated signaling and contraction in spontaneously hypertensive rat vascular smooth muscle cells. Circ Res 1999; 84: 505-515.

49 Ruperez M, Rodrigues-Diez R, Blanco-Colio LM, Sanchez-Lopez E, Rodriguez-Vita J, Esteban V, Carvajal G, Plaza JJ, Egido J, Ruiz-Ortega M. HMG-CoA reductase inhibitors decrease angiotensin II-induced vascular fibrosis: role of RhoA/ROCK and MAPK pathways. Hypertension 2007; 50: 377-383.

Supplementary Information accompanies the paper on Hypertension Research website (http://www.nature.com/hr) 